\title{
Diagnostic and clinical procedures of a patient with oral-facial-digital type II syndrome: a case report
}

\author{
Carla de Souza Lage ${ }^{1}$, Luis Candido Pinto da Silva ${ }^{2}$, Luciana Fonseca ${ }^{3}$, Roberval de Almeida Cruz ${ }^{4}$ \\ ${ }^{1}$ DDS. School of Dentistry, Pontifical Catholic University of Minas Gerais, Belo Horizonte, MG, Brazil. \\ ${ }^{2}$ DDS,MSD. Pediatric Dentistry Department, School of Dentistry, Pontifical Catholic University of Minas Gerais, Belo Horizon- \\ te, MG, Brazil. \\ ${ }^{3}$ DDS,MSD,PhD. Radiology Department School of Dentistry, Pontifical Catholic University of Minas Gerais, Belo Horizonte, \\ MG, Brazil. \\ ${ }^{4}$ DDS,MSD,PhD. Pediatric Dentistry Department, School of Dentistry, Pontifical Catholic University of Minas Gerais, Belo \\ Horizonte, MG, Brazil.
}

Correspondence:

Departamento de Odontologia - PUC Minas,

Av. D. José Gaspar, 500 - Prédio 46

30535-901 - Belo Horizonte - MG, Brazil.

E-mail:roberval@pucminas.br

Received: $18 / 10 / 2010$ Accepted: 17/12/2010

\author{
Lage CS, Silva LCP, Fonseca L, Cruz RA. Diagnostic and clinical proce- \\ dures of a patient with oral-facial-digital type II syndrome: a case report. J \\ Clin Exp Dent. 2011;3(3):e261-4. \\ http://www.medicinaoral.com/odo/volumenes/v3i3/jcedv3i3p261.pdf \\ Article Number: 50420 http://www medicinaoral.com/odo/indice.htm \\ (C) Medicina Oral S. L. C.I.F. B 96689336 - eISSN: 1989-5488 \\ eMail: jced@jced.es
}

\begin{abstract}
The oral-facial-digital syndromes (OFD) are part of a great number of genetic disorders. They are classified in categories based on characteristic abnormalities, including OFD type I (Papillon-Leage and Psaume syndrome) and OFD type II (Mohr syndrome). The aim of this article is to describe a clinical case of a patient with oralfacial-digital type II syndrome or Mohr syndrome, who underwent surgery for supernumerary teeth removal under endovenous sedation. Moreover, it is intended to discuss the importance of diagnostic and clinical procedures, with proper and concise indications about the limitations of patients with special needs treatment. Due to their clinical and physiological limitations, they must be treated by qualified professionals who are capable of performing various dental treatment procedures, including surgical removal of supernumerary teeth.
\end{abstract}

Key words: Oral-facial-digital type II, Mohr syndrome, supernumerary teeth, patients with special needs. 


\section{Introduction}

The oral-facial-digital syndromes (OFD) are part of a great number of genetic disorders. They are classified in some categories based on characteristic abnormalities, including OFD type I (Papillon-Leage and Psaume syndrome) and OFD type II (Mohr syndrome). More than nine different clinical entities have been defined. The different phenotypes can be explained by the wide variation in the genes expression (1-4).

The classification of OFD is based on clinical data and familial heritage, but there are reported cases which took into account additional traces of two or three OFDs $(5,6)$. The classification is also based on clinical phenotype and its heritage pattern. Despite the fact that the syndrome was primarily associated with a sex-linked sublethal recessive gene of an affected male patient whose siblings also showed the same syndrome, further study have demonstrated that it is a very rare disease and until now there is no many reports in the literature. Only less than 25 cases were described, most of them related with an autosomal recessive trait (7). The abnormalities detected in the patients include discrete lower stature, lower nasal bridge with displacement of the internal ocular angle, wide tip of the nose which is bifid in some patients, partial labial fissure in the midline, and hypertrophy of the lingual frenulum. Widening of the alveolar crest as well as hypoplasia of the crest of the zygomatic arch, and the body of the jaw can also be found. The conduction deafness apparently originates from of a defect of incus (8).

The Mohr syndrome is rarer than and is often confused with the OFD type I (3). However, OFD type II is a condition characterized by pre- and post-axial polidactyly of the hands and feet, partial hallux duplication, first metatarsal of the cuneiform and cuboid bones, relatively small hands with clinodactyly of the little finger, in addition to deafness (9). These are the main features that distinguish Mohr syndrome from OFD type I, in addition to the usual anomalies of the face and the mouth.

The diagnosis of OFDs can be rather confusing because many of the clinical characteristics of OFD type I and type II overlap (3). The autosomal recessive inheritance of OFD type II is the cardinal distinction between this syndrome and OFD type I (6). There is a possibility that the etiology of these syndromes could be explained by a genetic mutation with different degrees of expression. Since this syndrome has a 1 in 4 chance of recurrence, it is important to provide the patient's family with genetic counseling at the time of diagnosis (7).

Due to high variability in expression pattern, some of the abnormalities of the OFD syndromes are present in some patients but not others (10). Among these occasional abnormalities of OFD type II are: wormian skull bones, absence of central incisors, palatal cleft, multiple frenulum, pectus escavatum, scoliosis, mental retarda- tion, open bite, porencephaly, hydrocephaly, supernumerary teeth, congenital tongue lipoma and recurrent multiple keratogenic cysts (11-13).

The aim of this article is to describe a clinical case of a patient with OFD type II or Mohr syndrome who underwent a surgery for supernumerary tooth removal under endovenous sedation.

\section{Report of a Case}

A seven-year-old male patient, leukoderm, diagnosed with OFD type II of recessive autosomal etiology, as confirmed by a geneticist sought treatment at the Dental Clinic for Special Needs Patients. Examinations revealed neuro-motor-psychological deficiency. Data from computed tomography and encephalic magnetic resonance examination of the skull showed the presence of heterotopic lesions of the neurons in posterior ventricular walls. Hamartomas in the region of cinereal tuber (hypothalamus area) secondary to the bad formation of the central nervous system was also observed. However, it did not call for surgical intervention because they were not heterotopic neurons and they did not behave as expansive injuries.

The personal health history of this patient showed that during the pregnancy of his mother, prenatal control was carried out in accordance with medical orientation, without follow-up using imaging examinations. When the patient was three years old, he presented difficulties walking and convulsive crises. Further medical evaluation indicated that these symptoms were part of the clinical characteristics of the diagnosed syndrome. At that time the child was medicated with valproic acid and referred to the physical therapist. The condition of the child had improved after undergoing the prescribed treatment procedures at the time. The patient underwent surgery for adenoid removal under general anesthesia. A potent analgesic (paracetamol plus codeine) was prescribed post-surgery. However, the patient presented an allergic reaction with dermatological manifestations in the form of pruritus on the whole body. Therefore, he was medicated with anti-histamines.

The patient was initially examined by a pediatric dentist. During the first appointment, it was observed all the characteristic signs of the syndrome: lower and wider nasal bridge; external ocular angle tilted; hypertrophy of lingual frenulum; hypoplasia of the zygomatic arch and the body of the mandible; also movement alteration as well as polidactyly of the hands and feet and partial hallux duplication. Probably this was the etiologic factor for walking difficulties. Problems on speech were observed. At that time, the patient was under the supervision of a speech and occupational therapist. The procedures carried out at the time included dental prophylaxis, topical application of fluoride, dental hygiene and diet counseling. These simpler and non-invasive procedures aimed 

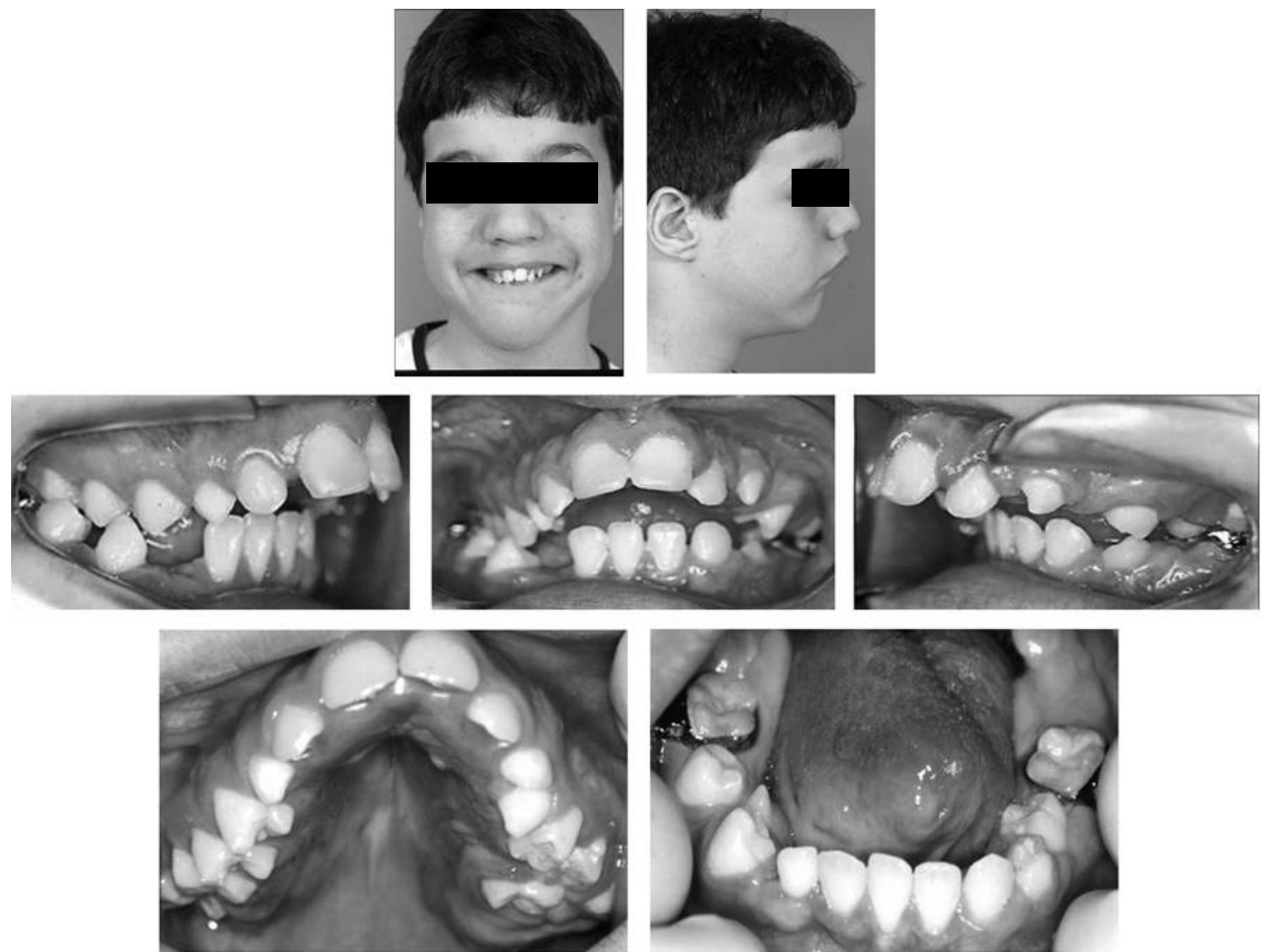

Fig. 1. Pretreatment facial and intraoral photographs. Observe the mandibular retraction

to improve the child's behavior in its initial resistance to the dental approach. After that, the patient was referred to an orthodontist for evaluation of his mandibular retrusion and lack of tooth alignment. Thus, it was decided to initiate the orthodontic treatment because the obser- ved malocclusion present by the patient. Metallic bands in both fist mandibular molars were cemented for using with a bumper (Fig. 1).

The panoramic x-ray (Fig. 2) revealed the presence of, at least, five supernumeraries teeth and signs of partial

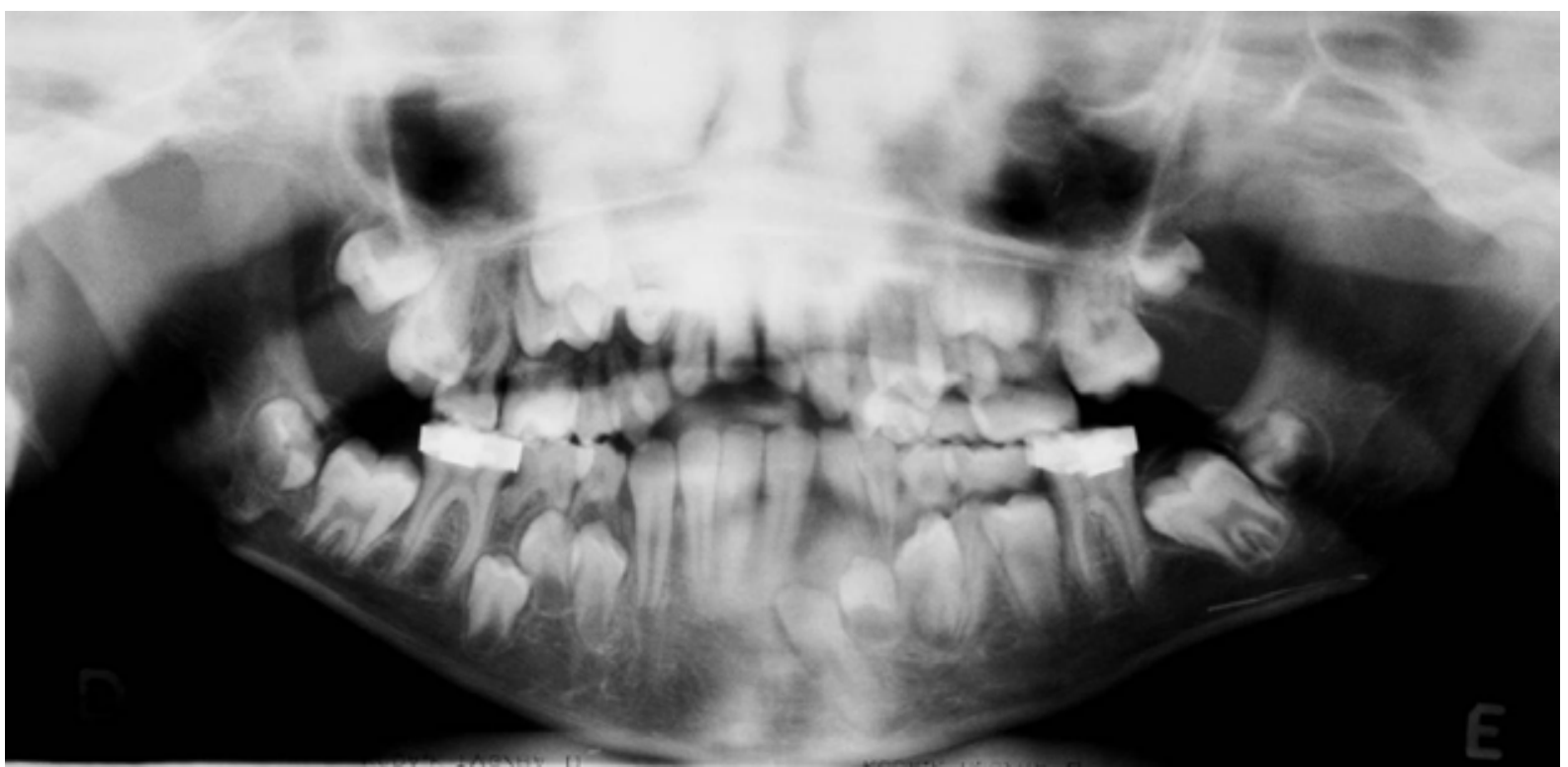

Fig 2. Pretreatment panoramic radiograph demonstrating presence of supernumerary teeth 
mineralization of the stylohyoid ligament. Moreover, the radiographic examination also showed the stage of dental formation of teeth $18,28,38$ and 48 . Teeth 17,12 , $22,24,25,27,35,34$ and 47 were in process of eruption. Teeth 15, 14, 13, 44 and 45 were in formation with a tendency to crowd. Tooth 23 was absent. Tooth 32 was badly positioned. The tooth 13 was located in transalveolar position. Deciduous teeth 53, 54, 55, 63, 75, 74, $73,72,84$ and 85 were detected.

To facilitate the orthodontic treatment supernumeraries teeth extraction were recommended. In accordance with the procedure complexity and the conditions of the patient, the surgical treatment was performed under endovenous sedation. The patient was examined by the medical anesthetist of the team and submitted to physical and laboratory examinations such as cardiovascular and pulmonary examinations. Complementary examinations for evaluation of the number of platelets, red and white blood cells, glycemia, creatinine dosage for verification of the renal function, bleeding and coagulation times, and activation time of partial thrombin were performed. The two upper supernumeraries teeth were located in the left and right palatal side, in the premolar area. In the mandible, the two supernumeraries teeth from the left side were in the lingual position and one from the right side were positioned buccally also in the premolar area. They were surgically extracted.

The informed consent was obtained from the mother of the patient prior to its dental treatment.

\section{Discussion}

Patients with OFDs are considered patients with special needs. Due to their clinical and physiological limitations, they must be treated by qualified professionals who are capable of performing various dental treatment procedures (14).

Endovenous sedation has precise indications and does not have to be used routinely in normal patients (15). Moreover, it is important to examine the condition of the patient prior to the operation and constantly monitor him during the anesthesia and surgical procedures (16).

The majority of patients with OFD type II possess common abnormalities that complicate the diagnosis and the differentiation between OFD types I and II. It is necessary to recognize the oral, facial, and digital characteristics of this specific group of patients in order to provide them with the best treatment $(17,18)$.

As general characteristics, the patient presented slightly lower stature, conductive deafness and apparent involvement of the central nervous system that resulted in difficulties in speech and locomotion, visible signs during the physical examination. The central nervous system involvement is considered rare in this disease. The patient, by their socio-economic status, had no access to prenatal care and there was no condition to establish the etiology of this abnormality. On the other side he displays normal intelligence.

In this reported case, a large number of supernumerary teeth was observed in the patient and the teeth were removed surgically. Nowadays, the patient is undergoing orthodontic treatment to improve the dental alignment and occlusion.

\section{References}

1. Papillon-Leage, Psaume J. Hereditary abnormality of the buccal mucosa: abnormal bands and frenula. Revue Stomatol. 1954;55:209-27.

2. Toriello HV. Heterogeneity and variability in the oral-facial-digital syndromes. Am J Med Genet Suppl. 1988;4:149-59.

3. Toriello HV. Oral-facial-digital syndromes, 1992. Clin Dysmorphol. 1993;2:95-105.

4. Prpic I, Cekada S, Franulovic J. Mohr syndrome (oro-facial-digital syndrome II) - a familial case with different phenotypic findings. Clin Genet. 1995;48:304-7.

5. Chitayat D, Stalker HJ, Azouz EM. Autosomal recessive oral-facialdigital syndrome with resemblance to OFD types II, III, IV and VI: a new OFD syndrome? Am J Med Genet. 1992;44:567-72.

6. Steichen-Gersdorf E, Gassner I, Covi B, Fischer H. Oral-facial-digital syndrome II. Transitional type between Mohr and the Majewski syndrome: report of a new case with congenital stenosis of the trachea. Clin Dysmorphol. 1994:3:245-50.

7. Hosalkar HS, Shah H, Gujar P, Kulkarni A, Yagnik MG. Mohr syndrome: a rare case and distinction from orofacial digital syndrome I. J Postgrad Med. 1999;45:123-4.

8. Baraitser M. The orofaciodigital (OFD) syndromes. J Med Genet. 1986;23:116-9.

9. Gurrieri F, Franco B, Toriello H, Neri G. Oral-facial-digital syndromes: review and diagnostic guidelines. Am J Med Genet A. 2007;143A:3314-23.

10. Rimoin DL, Edgerton MT. Genetic and clinical heterogeneity in the oral-facial-digital syndromes. J Pediatr. 1967;71:94-102.

11. Sakai N, Nakakita N, Yamazaki Y, Ui K, Uchinuma E. Oral-facialdigital syndrome type II (Mohr syndrome): clinical and genetic manifestations. J Craniofac Surg. 2002;13:321-6.

12. Ghossaini SN, Hadi U, Tawil A. Oral-facial-digital syndrome type II variant associated with congenital tongue lipoma. Oral Surg Oral Med Oral Pathol Oral Radiol Endod. 2002;94:324-7.

13. Lindeboom JA, Kroon FH, de Vires J, van den Akker HP. Multiple recurrent and odontogenic keratocysts associated with oral-facialdigital syndrome. Oral Surg Oral Med Oral Pathol Oral Radiol Endod. 2003;95:458-62.

14; Crall JJ. Improving oral health for individuals with special health care needs. Pediatr Dent. 2007;29:98-104.

15. Morales-Chávez MC. Dental management of a patient with Rubinstein Taybi syndrome. Spec Care Dentist. 2010;30:124-6.

16. Lourenço-Matharu L, Roberts GJ. Oral sedation for dental treatment in young children in a hospital setting. Br Dent J. 2010;209(7):E12.

17. Velepic MS, Sasso A, Velepic MM, Lustica I, Starcevic R, Komljenovic D. Combined anomalies of the palate in Mohr syndrome: is preoperative electromyography of the palate useful? J Pediatr Surg. 2004;39:220-2.

18. Biswas A, Ghosh JK, Sinha MK, Basu K, Chatterjee S. MohrClaussen syndrome or oral-facial-digital syndrome (OFDS) type-II. J Pak Med Assoc. 2009;59:484-6. 